Relations industrielles

Industrial Relations

\title{
Entreprises d'intérêt collectif et création d'emplois. Initiatives locales de création d'emplois. OCDE, Paris, 1984, 77 pp., ISBN \\ 92-64-22569-2
}

\section{Louis Gosselin}

Volume 41, numéro 1, 1986

URI : https://id.erudit.org/iderudit/050189ar

DOI : https://doi.org/10.7202/050189ar

Aller au sommaire du numéro

Éditeur(s)

Département des relations industrielles de l'Université Laval

ISSN

0034-379X (imprimé)

1703-8138 (numérique)

Découvrir la revue

Citer ce compte rendu

Gosselin, L. (1986). Compte rendu de [Entreprises d'intérêt collectifet création d'emplois. Initiatives locales de création d'emplois. OCDE, Paris, 1984, 77 pp., ISBN 92-64-22569-2]. Relations industrielles / Industrial Relations, 41(1), 189-190. https://doi.org/10.7202/050189ar

Tous droits réservés @ Département des relations industrielles de l'Université Laval, 1986
Ce document est protégé par la loi sur le droit d'auteur. L’utilisation des services d’Érudit (y compris la reproduction) est assujettie à sa politique d'utilisation que vous pouvez consulter en ligne.

https://apropos.erudit.org/fr/usagers/politique-dutilisation/ 


\section{RECENSIONS}

\section{BOOK REVIEWS}

Entreprises d'intérêt collectif et création d'emplois. Initiatives locales de création d'emplois, OCDE, Paris, 1984, 77 pages, ISBN 92-64-22569-2

La crise économique vécue par les pays industrialisés au début des années 80 a rendu plus visibles les inégalités d'emplois. Certains gouvernements ont tenté d'apporter des mesures correctives appropriées.

Ce livre écrit par madame Jocelyne Gaudin, chargée de recherches au centre «Travail et Société» de l'Université de Paris IX - Dauphine pour le compte de l'Organisation de coopération et de développement économiques (OCDE), prend pour hypothèse que la création d'entreprises d'intérêt collectif (E.I.C.) peut être une voie souhaitable dans la recherche de nouvelles possibilités d'emplois.

Ce rapport fait état d'actions non concertées de certains pays industrialisés qui tentent d'utiliser un secteur intermédiaire de l'économie de marché entre le public et le privé. On retrouve dans ce secteur certains types d'organisations hétéroclites qui poursuivent avec différentes intensités des objectifs sociaux, culturels et économiques. Un point commun les unit: la création d'emplois.

Appuyé par des notes abondantes tirées d'auteurs provenant de pays industrialisés, tels que le Canada, l'Angleterre, la France, l'Allemagne et les États-Unis, ce document fait ressortir l'importance de ces nouvelles structures dans une société industrielle en changement.

L'ouvrage se divise en trois parties. Dans le chapitre I, l'auteure établit une typologie des entreprises d'intérêt collectif à partir de stratégies de création d'emplois.

Ces types d'entreprises qu'elle classifie en trois groupes (travail social, entreprise communautaire et fonctionnement collectif) sont au prise avec une dualité d'objectif: le profit et la création d'emplois.

Que ces E.I.C. naissent sous l'impulsion des travailleurs sociaux afin de faciliter l'accès a l'emploi à des travailleurs marginaux, qu'elles apparaissent sous forme coopérative en réponse à un désir du milieu de se prendre en main ou se développent sous l'impulsion de communautés isolées, ces structures font face à des obstacles majeurs dans une société qui n'a pas prévu de place pour ces formes d'organisations.

Le chapitre II identifie d'une façon claire et concise des obstacles et facteurs de diffusion de ce genre d'entreprises. Ces facteurs sont d'ordre juridique et financier. Juridique, dans le sens que les pays industrialisés ont peine à situer ce genre d'organisation préoccupée autant du social que de l'économique. Financier, dans le sens où les responsables du financement public et privé doutent de la crédibilité de ces formes de structures. De plus, centralisés au niveau des décisions, les fournisseurs de capitaux ne sont pas aptes a juger de la valeur des projets.

Ce chapitre se termine par une évaluation sommaire des programmes gouvernementaux appliqués au Québec. Les conclusions très positives de cette évaluation gagneraient à être nuancées. 
Enfin, en conclusion dans le chapitre III, l'auteure identifie des incitations publiques qui pourraient favoriser l'émergence et le développement des entreprises d'intérêt collectif afin qu'elles puissent maximiser leur chance de réussite et acquérir une plus grande crédibilité dans le milieu.

Ce rapport, bien que succinct, décrit assez bien cette nouvelle forme d'entreprises d'intérêt collectif qui se développe de plus en plus dans les pays industrialisés. Il n'apporte pas toutes les nuances souhaitées et son ampleur ne permet pas une analyse très poussée de ce phénomène.

Louis GOSSELIN

Université du Québec à Rimouski

La gestion du personnel: aspects juridiques, tome I (recrutement, carrière, mouvements de personnel), $2^{\mathrm{e}}$ édition, par Michel Despax et Jean Pélissier, Paris, Éditions Cujas, 1984, 400 pp., ISBN 2-2548-4031-0

Cet ouvrage, en deux tomes, dont seul le premier a paru au moment où j'écris, remplace celui conçu en trois volumes il y a une dizaine d'années, et qui avait, dès l'époque, rendu un insigne service aux responsables du personnel. Il figure désormais parmi les références indispensables aux praticiens de la Fonction, et à ceux qui aspirent à le devenir, et ne devrait pas laisser indifférents, plus largement, les observateurs des relations industrielles tout court.

Ceux-ci noteront, dès l'avant-propos, que les auteurs, juristes réputés, déplorent à juste titre qu'au «travail en miettes» dans le monde industriel correspond une dispersion des connaissances relatives au travail, peu propice aussi bien à la réflexion féconde qu'à l'action efficace», "balkanisation du savoir (...) de nature à désorienter le praticien appelé à mettre en oeuvre, au niveau des entreprises, une politique de personnel dont la cohérence est une condition essentielle d'efficacité».

Soucieux de voir prises en compte les incidences d'ordre juridique des décisions dans le domaine du personnel, les règles juridiques qui président à maintes opérations ayant trait au travail, M. Despax et J. Pélissier précisent qu'ils n'ont point la prétention de remplacer dans leur domaine les travaux des spécialistes des matières connexes, mais uniquement le souhait de faire oeuvre juridique "dans une perspective qui se veut pluridisciplinaire, pour des praticiens d'entreprise, en «fonction directe des besoins et des préoccupations des directeurs du personnel».

Il est caractéristique, et encourageant, que dès le début du chapitre I (du titre I sur «les problèmes de recrutement du personnel»), les auteurs déplorent l'absence dans nombre d'entreprises d'une planification des ressources humaines - laquelle, à l'heure qu'il est, chemine, dans les collectivités les plus avancées, vers la planification stratégique - , intégrée dans la planification générale de l'entreprise, et, plus particulièrement, ce mode d'approche logique de la gestion des ressources humaines, consistant dans la projection à moyen et à long terme des besoins et des ressources en personnel, qu'est la gestion prévisionnelle.

Guidés, tout au long du livre, par la préoccupation du «comment faire», ils abordent, successivement, les relations avec les services de l'emploi, les techniques de sélection, le choix du type de contrat (à durée déterminée ou indéterminée) - thème toujours d'actualité dans le débat professionnel et politique en France, l'élaboration du contrat et les responsabilités en matière de recrutement. Le chapitre II aborde les problèmes particuliers de recrutement, dont le recours aux entreprises de travail temporaire, particulièrement visées par une ordonnance de 1982 qui a assaini le marché, et de nouveau en déploiement, modéré, depuis peu. 